\title{
Smart Wireless Railway Monitoring System
}

\author{
K. Liu*, W.H. Siew*, R.W. Stewart*, Y. Wang ${ }^{\dagger}$ \\ *Department of Electronic and Electrical Engineering, University of Strathclyde, Glasgow G1 1XW, UK \\ ${ }^{+}$Now an Electronic Systems Design Consultant in China
}

Keywords: Wireless, time synchronization.

\begin{abstract}
With the increased demand for railway services, train speed and density are consistently increasing in the last two decades. As a result, more strict safety requirements for railway signaling, control and infrastructure are needed. Accompanying that trend, in recent years, wireless communication techniques have also advanced rapidly. Especially with smart low cost wireless communication techniques like WIFI, Bluetooth, and ZigBee going into maturity, thus making it possible to develop a wireless system to monitor a railway's signaling or control or infrastructure condition. An effective low cost monitor system will help the normal function of railway systems. This paper gives a description of the development of one such monitoring system.
\end{abstract}

The whole system consists of a remote controller and many monitor units. The remote controller gets data from the monitor units and sends control commands and monitoring control information to the monitor units. The paper will show how such a basic set up could be deployed for railway monitoring using wireless technology.

\section{Introduction}

With the increasing demand for railway services, train-speed and line-occupation-density have increased in the last two decades. As a result, more strict safety requirements for railway signalling, control and infrastructure are needed. Accompanying that trend, in recent years, the wireless communication techniques have also advanced significantly, especially smart low cost wireless communication techniques going into maturity and thus making it possible to develop a wireless system to monitor the condition of a railway's signalling or control system or infrastructure. An effective low cost monitoring system will help the normal function of a railway system. This paper gives a description of the development of one such monitoring system.

Conventional methods of railroad maintenance and safety assurance are based on separate periodical inspections of track and equipment [1]. These methods have two limitations: firstly they leave many defects undetected for a relatively long time. Secondly, it is difficult to combine the data from track inspection and equipment inspections. This system described in this paper aims to solve these two limitations by introduce a solution which nearly real time simultaneously inspects both vehicles and infrastructure.

\section{Proposed system structure}

The whole system consists of one remote controller and many monitor units. The Remote controller is a computer incorporated with a transceiver. The remote controller communicates with the nearest monitor unit and it is through this communication that an operator is able to get data from all monitor units and sends control commands and monitoring or control information to the monitor units. Monitor units are used to collect all kinds of information on track condition and signalling.

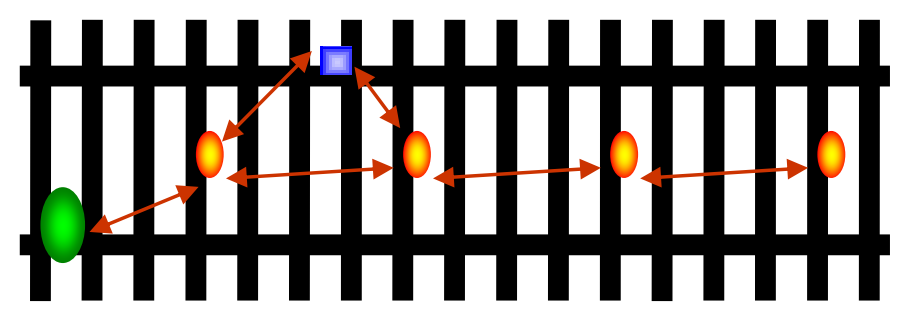

Remote controller $\square_{\text {locomotive }} \mathrm{O}$ Static monitor unit Fig1 System structure

There are two kinds of monitor units: one is static and each is distributed along the railway, has determined locations and exists all the time in the network. The other is dynamic and the unit is installed on the train locomotive, so that it "joins" the network dynamically and changes their locations continuously. Every unit has its unique identity and has both data acquisition function and data communication function.

In addition, the static monitor units could only communicate with their neighbouring static units and neighbouring dynamic units. So if one unit would like to send some data back to the remote controller, it has to use the static monitor units between them as relays.

Based on need, the wagons could also have some monitor units installed on them, which together with the locomotive monitor unit form a small local system which communicate with the outside world through the locomotive. They would work in a much similar way as the trackside-system: the locomotive works as control node; the wagons work as slave nodes which just can communicate with the two neighbouring 
wagons; the midway wagons work also as relays of information.

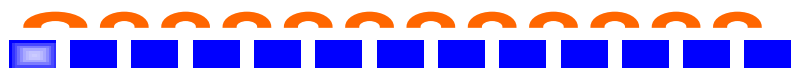

$\square$ Locomotive $\square$ Wagon Communications

Fig2 train local system

To save power the system works in TDMA mode. For static monitor units, the working time is divided into three time slots: Transmitting slot, Receiving slot, and Dynamic slot. In the "transmit slot", the static unit could transmit acquired data to its neighbouring unit, which at the same time should be in "receive slot". In fact the first frame of data from the transmitter will tell the receiver how many frames to expect. So after the data exchange is finished both units could go to sleep mode and wake up at the beginning of the next timeslot. In addition, when one static unit is in transmitting slot its two neighbouring units must be in receiving slot, and vice verse. The dynamic slot is specially reserved for the static unit to communicate with its neighbouring dynamic unit if there is one. For dynamic units, normally it will stay in receiving mode so it can listen to the static monitor units and know when they are in dynamic slot so that data exchange could be carried out.

\section{Time Synchronization}

Time Synchronization is the basis for a TDMA system. Without synchronization, devices do not know when to enter which slots. Besides that, it is helpful for the correct understanding of time sequence of events on different nodes and thus could be used to increase energy efficiency. For example it will allow the nodes to sleep for a given time and then wake up at the correct time to wait for possible signals from other nodes. This characteristic is very desirable for our system since the static monitor node is normally battery powered, but rechargeable. The life of the battery is mostly determined by time durance in which the unit is active.

\subsection{Time synchronization on static monitor units}

The static monitor units are usually powered by batteries. Therefore, power efficiency is of high priority. Aiming to be a low cost and low power solution, the static monitor should implement a wireless time synchronization protocol based on current data communication hardware instead of using GPS which will add more cost and more power consumption.

In common wide-area wireless distributed network, wireless time synchronization is usually implemented in a multi-hop fashion, where the synchronization is done one loop after another loop until the last one [2]. Our system is very different, i.e. it is one-dimensional. In other words, it covers one long line instead of one big area. If we use the multi-hop strategy, we will make too many hops. For example, if we have 1000 static units, which are located every 100 meters along the railway. If 200 meters are equivalent to one hop, we need 500 hops. Assuming that every hop needs $1 \mathrm{~ms}$ to achieve synchronization, we need to do, in total, 500 times synchronization and need at least $500 \mathrm{~ms}$ to finish the whole synchronization process. Therefore, it is not suitable for our system.

There is a way to greatly decrease the synchronization time, which is based on the fact that it is the remote controller that needs to know the correct time differences not the static monitor units. In fact the static monitor units do not need to be directly synchronized to the remote controller, they just need to know their relative time to the neighbour units. So if each unit knows its relative time to its neighbour, under the condition that the first static monitor unit know its relative time to the remote controller, we can clearly derive the correct time differences between the static units with the remote controller and carries on the compensation at the control panel.

An example of the synchronization process is given below. Static monitor units and the remote controller are divided into two groups $\mathrm{N}_{2 \mathrm{n}}, \mathrm{N}_{2 \mathrm{n}+1}$ according to the even and odd sequence from the remote controller. The remote controller is of number zero $\left(\mathrm{N}_{0}\right)$. Firstly, the even group members, $\mathrm{N}_{2 \mathrm{n}+1}$ does synchronization with the neighboring $\mathrm{N}_{2 n}$, by which the time difference between $\mathrm{N}_{2 n+1}$ and $\mathrm{N}_{2 n}, \mathrm{t}_{(2 \mathrm{n}+1)-2 \mathrm{n}}$ is known. Secondly, $\mathrm{N}_{2 \mathrm{n}+1}$ does synchronization with the neighboring $\mathrm{N}_{2 \mathrm{n}+2}$, by which the time difference between $\mathrm{N}_{2 \mathrm{n}+1}$ and $\mathrm{N}_{2 \mathrm{n}+2, \mathrm{t}}$ $(2 n+2)-(2 n+1)$ is known. So by adding up all the time difference before it, the time difference between the static monitor unit and the remote controller is known. Taking the same 1000 static monitor units example, the whole process needs to do the same number of synchronizations. But by doing them in parallel, the total synchronization time is just two synchronization durations, namely $2 \mathrm{~ms}$, which is just $1 / 500$ of the initial synchronization time.

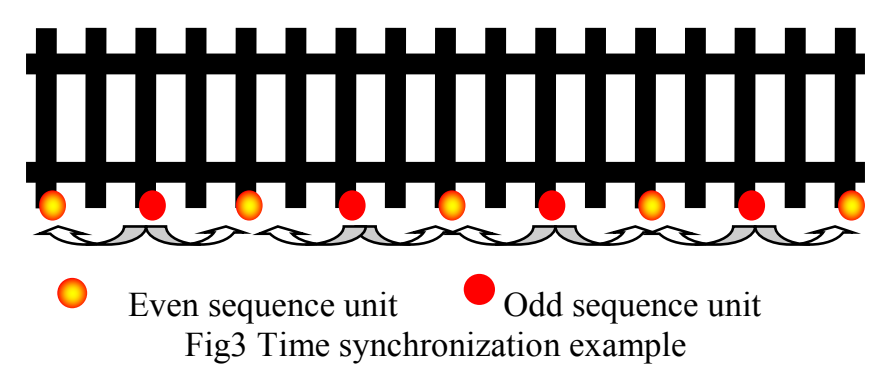

There is one concern with time synchronization: clock drift, which is caused by crystal aging and other environmental factors such as temperature variation. In our system, the clock drift problem is addressed by periodically broadcasting synchronization message. Thus, the relative clock drift could be tracked.

\subsection{Time synchronization on dynamic monitor units}

Since dynamic monitor units are installed on trains, especially on passenger trains where there is usually enough power, they could be in active mode all the time and could use GPS as a time synchronization source. If not convenient or not enough 
power is available; the dynamic units could work in a similar way to the static units. Under this situation, the locomotive should have GPS receiver installed and work as local remote controller.

\section{Hardware structure}

Every monitor unit consists of two modules, data acquisition module and communication and control module. In the data acquisition module, there would be some sensors connected to a programmable device, through which data from the sensors would be saved into memory. When something abnormal happens, the related data would be sent to the remote controller by the communication module. Inside the communication and control module, there is a transceiver for data communication and for time synchronization.

Fig. 4 is the block diagram for the dynamic monitor unit. The static monitor unit has a similar structure except for the absence of a GPS receiver. In some situations, when not many sensors are required, the programmable device could also be removed.

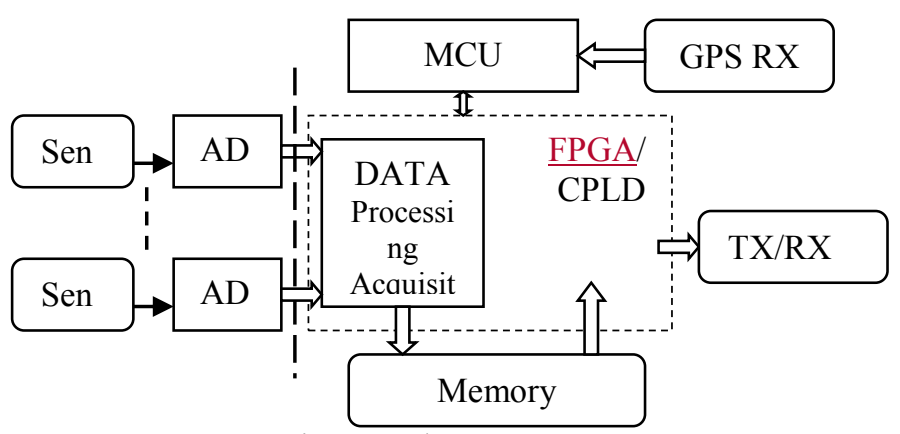

Fig4, Hardware structure

\section{Conclusion}

This paper introduced a low cost system for railway monitoring. The system deals with the condition monitoring of both the infrastructure and trains. A simple and effective time synchronization method for railway application is also described.

\section{References}

[1] Nejikovsky, B.; Keller, E. "Wireless communications based system to monitor performance of railvehicles", Railroad Conference, 2000. Proceedings of the 2000 ASME/IEEE Joint Volume , Issue , 2000 Page(s):111 124

[2] S. Ganeriwal, R. Kumar, M. B. Srivastava, "Timingsync protocol for sensor networks," ACM Conference on Embedded Networked Sensor Systems (SENSYS 2003). Pages: 138 - 149 (2003) 